\title{
Supracerebellar transtentorial approach to the tentorial incisura and beyond
}

\author{
Kyle I. Swanson, MD, Ulas Cikla, MD, Kutluay Uluc, MD, and Mustafa K. Baskaya, MD \\ University of Wisconsin, Department of Neurological Surgery, Madison, Wisconsin \\ The supracerebellar transtentorial approach via a suboccipital craniotomy provides a corridor to reach lesions of the \\ tentorial incisura and supratentorial lesions of the posterior medial basal temporal lobe, such as lesions of the posterior \\ parahippocampal and fusiform gyri. The supracerebellar transtentorial approach obviates the need for either retraction \\ of eloquent cortex or a transcortical route to reach lesions in this region. We present three cases that demonstrate the \\ utility of this approach: a left-sided tentorial meningioma with superior projection, a left-sided posterior parahippocampal \\ cavernous malformation, and a left-sided posterior parahippocampal grade 2 oligodendroglioma.
}

The video can be found here: https://youtu.be/OLnzUGZfUqk.

KEYWORDS parahippocampal gyrus; supracerebellar transtentorial approach; tentorial meningioma; video 